KYUNGPOOK Math. J. 52(2012), 39-47

http://dx.doi.org/10.5666/KMJ.2012.52.1.39

\title{
Further Results about the Normal Family of Meromorphic Functions and Shared Sets
}

\author{
JIANMING QI* \\ Department of Mathematics and Physics, Shanghai Dianji University, Shanghai, \\ 200240, China \\ e-mail : qijianmingdaxia@163.com \\ GuOWEI ZHANG \\ School of Mathematics and Statistics, Anyang Normal University, Anyang, 455002, \\ China \\ e-mail : zhirobo@gmail.com

\section{LINLIN ZHOU} \\ Department of Mathematics, Zhenjiang watercraft college 212003, China \\ e-mail : zhoulinlin928@126.com \\ Abstract. Let $\mathcal{F}$ be a family of meromorphic functions in a domain $D$, and let $k, n(\geq 2)$ \\ be two positive integers, and let $S=\left\{a_{1}, a_{2}, \ldots, a_{n}\right\}$, where $a_{1}, a_{2}, \ldots, a_{n}$ are distinct finite \\ complex numbers. If for each $f \in \mathcal{F}$, all zeros of $f$ have multiplicity at least $k+1, f$ and \\ $G(f)$ share the set $S$ in $D$, where $G(f)=P\left(f^{(k)}\right)+H(f)$ is a differential polynomial of $f$, \\ then $\mathcal{F}$ is normal in $D$.
}

\section{Introduction}

Let $\mathcal{C}$ be the whole complex domain. Let $D$ be a domain in $\mathcal{C}$ and $\mathcal{F}$ a family of meromorphic functions defined in $D$. $\mathcal{F}$ is said to be normal in $D$, in the sense of Montel, if each sequence $\left\{f_{n}\right\} \subset \mathcal{F}$ has a subsequence $\left\{f_{n_{j}}\right\}$ which converse spherically locally uniformly in $D$, to a meromorphic function or $\infty$. (see [3]).

Let $f$ and $g$ be meromorphic functions on a domain $D$, and let $a$ and $b$ be two complex numbers. If $g(z)=b$ whenever $f(z)=a$, we write

$$
f(z)=a \Rightarrow g(z)=b
$$

* Corresponding Author.

Received March 3, 2010; revised December 11, 2010; accepted September 23, 2011.

2010 Mathematics Subject Classification: 30D35.

Key words and phrases: Meromorphic functions, Nevanlinna theory, Normal family, Share value. 
If $f(z)=a \Rightarrow g(z)=b$ and $g(z)=b \Rightarrow f(z)=a$, we write

$$
f(z)=a \Leftrightarrow g(z)=b .
$$

If $f(z)=a \Leftrightarrow g(z)=a$, we say that $f$ and $g$ share $a$ on $D$.

Let $a_{i}(z),(i=1,2, \ldots, q-1), b_{j}(z),(j=1,2, \ldots, n)$ be analytic in $D$, $n_{0}, n_{1}, \ldots, n_{k}$ be non-negative integers. $q$ be a positive integer. Set

$$
\begin{gathered}
P(\omega)=\omega^{q}+a_{q-1}(z) \omega^{q-1}+\ldots+a_{1}(z) \omega, \\
M\left(f, f^{\prime}, \ldots, f^{(k)}\right)=f^{n_{0}}\left(f^{\prime}\right)^{n_{1}} \ldots\left(f^{(k)}\right)^{n_{k}}, \\
\gamma_{M}=n_{0}+n_{1}+\ldots+n_{k}, \\
\Gamma_{M}=n_{0}+2 n_{1}+\ldots+(k+1) n_{k} .
\end{gathered}
$$

$M\left(f, f^{\prime}, \ldots, f^{(k)}\right)$ is called the differential monomial of $F, \gamma_{M}$ the degree of $M\left(f, f^{\prime}, \ldots, f^{(k)}\right)$ and $\Gamma_{M}$ the weight of $M\left(f, f^{\prime}, \ldots, f^{(k)}\right)$.

Let $M_{i}\left(f, f^{\prime}, \ldots, f^{(k)}\right),(i=1,2, \ldots, n)$ be differential monomials of $f$. Set

$$
\begin{gathered}
H\left(f, f^{\prime}, \ldots, f^{(k)}\right)=b_{1}(z) M_{1}\left(f, f^{\prime}, \ldots, f^{(k)}\right)+\ldots+b_{n}(z) M_{n}\left(f, f^{\prime}, \ldots, f^{(k)}\right), \\
\gamma_{H}=\max \left\{\gamma_{M_{1}}, \gamma_{M_{2}}, \ldots, \gamma_{M_{n}}\right\}, \\
\Gamma_{H}=\max \left\{\Gamma_{M_{1}}, \Gamma_{M_{2}}, \ldots, \Gamma_{M_{n}}\right\} .
\end{gathered}
$$

$H\left(f, f^{\prime}, \ldots, f^{(k)}\right)$ is called the differential polynomial of $f, \gamma_{H}$ the degree of $H\left(f, f^{\prime}, \ldots, f^{(k)}\right)$ and $\Gamma_{H}$ the weight of $H\left(f, f^{\prime}, \ldots, f^{(k)}\right)$.

Set

$$
\begin{aligned}
\left.\frac{\Gamma}{\gamma}\right|_{H} & =\max \left\{\frac{\Gamma_{M_{1}}}{\gamma_{M_{1}}}, \frac{\Gamma_{M_{2}}}{\gamma_{M_{2}}}, \ldots, \frac{\Gamma_{M_{n}}}{\gamma_{M_{n}}}\right\}, \\
G(f) & =P\left(f^{(k)}\right)+H\left(f, f^{\prime}, \ldots, f^{(k)}\right) .
\end{aligned}
$$

Schwick [7] discovered a connection between normality criteria and shared values and proved

Theorem A. Let $\mathcal{F}$ be a family of meromorphic functions in a domain $D$, and a, $b, c$ be distinct complex numbers, If for each $f \in \mathcal{F}, f$ and $f^{\prime}$ share $a, b, c$, then $\mathcal{F}$ is normal in $D$.

Pang and Zalcman [6] improved Theorem A as follows.

Theorem B. Let $\mathcal{F}$ be a family of meromorphic functions in a domain D, and let $a, b$ be two distinct complex numbers. If, for each $f \in \mathcal{F}, f$ and $f^{\prime}$ share $a$ and $b$ in $D$, then $\mathcal{F}$ is normal in $D$.

In 2002, Fang and Zalcman [2] proved

Theorem C. Let $\mathcal{F}$ be a family of meromorphic functions in a domain D, let a be nonzero finite complex number, and let $k$ be a positive integer. If, for each $f \in \mathcal{F}$, 
all zeros of $f$ have multiplicity at least $k+1$, and $f$ and $f^{(k)}$ share $a$ in $D$, then $\mathcal{F}$ is normal in $D$.

Let $S$ be a set of complex numbers. If $f(z) \in S$ if and only if $g(z) \in S$ in a domain $D$, then we say $f$ and $g$ share the set $S$ in D.

It is natural to ask that that whether Theorem $\mathrm{C}$ is valid or not if $f$ and $f^{(k)}$ share a value $a$ was replaced by $f$ and $f^{(k)}$ share a set $S$ ?

Recently, Lei, Fang and Yang [4] proved:

Theorem D. Let $\mathcal{F}$ be a family of meromorphic functions in a domain $D$, let $n(\geq 2), k$ be two positive integers, and let $S=\left\{a_{1}, a_{2}, \ldots, a_{n}\right\}$, where $a_{1}, a_{2}, \ldots, a_{n}$ are distinct finite complex numbers. If for each $f \in \mathcal{F}$, all zeros of $f$ have multiplicity at least $k+1$, and $f$ and $f^{(k)}$ share the set $S$ in $D$, then $\mathcal{F}$ is normal in $D$.

Theorem E. Let $\mathcal{F}$ be a family of meromorphic functions in a domain $D$, let $n, m, k$ be three positive integers, and let $S=\left\{a_{1}, a_{2}, \ldots, a_{n}\right\}, S_{2}=\left\{b_{1}, b_{2}, \ldots, b_{m}\right\}$ where $a_{1}, a_{2}, \ldots, a_{n}, b_{1}, b_{2}, \ldots b_{m}$ are distinct finite complex numbers. If for each $f \in \mathcal{F}$, all zeros of $f$ have multiplicity at least $k$, and $f$ and $f^{(k)}$ share the sets $S_{1}$ and $S_{2}$ in $D$, then $\mathcal{F}$ is normal in $D$.

In [4], Lei, Fang and Yang give the examples to show all zeros of $f$ have multiplicity are best possible in the above theorems.

In this paper, we extend Theorem D and Theorem $\mathrm{E}$ as follows.

Theorem 1. Let $\mathcal{F}$ be a family of meromorphic functions in a domain $D$, let $n(\geq 2), k$ be two positive integers, and let $S=\left\{a_{1}, a_{2}, \ldots, a_{n}\right\}$, where $a_{1}, a_{2}, \ldots, a_{n}$ are distinct finite complex numbers. If for each $f \in \mathcal{F}$, all zeros of $f$ have multiplicity at least $k+1$, and $f$ and $G(f)$ share the set $S$ in $D$, where $G(f)=P\left(f^{(k)}\right)+H(f)$ be a differential polynomial of $f$ satisfying $q \geq \gamma_{H}$, and $\left.\frac{\Gamma}{\gamma}\right|_{H}<k+1$, then $\mathcal{F}$ is normal in $D$.

Theorem 2. Let $\mathcal{F}$ be a family of meromorphic functions in a domain $D$, let $n, m, k$ be three positive integers, and let $S_{1}=\left\{a_{1}, a_{2}, \ldots, a_{n}\right\}, S_{2}=\left\{b_{1}, b_{2}, \ldots, b_{m}\right\}$ where $a_{1}, a_{2}, \ldots, a_{n}, b_{1}, b_{2}, \ldots b_{m}$ are distinct finite complex numbers. If for each $f \in \mathcal{F}$, all zeros of $f$ have multiplicity at least $k$, and $f$ and $P\left(f^{(k)}\right)$ share the sets $S_{1}$ and $S_{2}$ in $D$, where $a_{i}(z)$ in $P(f)$ are constants, then $\mathcal{F}$ is normal in $D$.

By Theorem 1, we immediately deduce

Corollary 1. Let $\mathcal{F}$ be a family of meromorphic functions in a domain $D$, let $n(\geq 2), k$ be two positive integers, and let $S=\left\{a_{1}, a_{2}, \ldots, a_{n}\right\}$, where $a_{1}, a_{2}, \ldots, a_{n}$ are distinct finite complex numbers. If for each $f \in \mathcal{F}$, all zeros of $f$ have multiplicity at least $k+1$, and $f$ and $L(f)$ share the set $S$ in $D$, where $L(f)=f^{(k)}+a_{1}(z) f^{(k-1)}+$ $a_{2}(z) f^{(k-2)}+, \ldots,+a_{k-1}(z) f^{\prime}+a_{k}(z) f$ and $a_{i}(z),(i=1,2, \ldots, k)$ are analytic in $D$, then $\mathcal{F}$ is normal in $D$.

By Theorem 2, we immediately deduce 
Corollary 2. Let $\mathcal{F}$ be a family of meromorphic functions in a domain $D$, let $n, m, k$ be three positive integers, and let $S_{1}=\left\{a_{1}, a_{2}, \ldots, a_{n}\right\}, S_{2}=\left\{b_{1}, b_{2}, \ldots, b_{m}\right\}$ where $a_{1}, a_{2}, \ldots, a_{n}, b_{1}, b_{2}, \ldots b_{m}$ are distinct finite complex numbers. If for each $f \in \mathcal{F}$, all zeros of $f$ have multiplicity at least $k$, and $f$ and $\left(f^{(k)}\right)^{q}$ share the set $S_{1}$ and $S_{2}$ in $D$, where $q$ is a positive integer, then $\mathcal{F}$ is normal in $D$.

\section{Preliminaries and lemmas}

In order to prove our results, we need the following lemmas.

Lemma 2.1[6,9]. Let $\mathcal{F}$ be a family of meromorphic functions in the unit disc $\triangle$ with the property that for each $f \in \mathcal{F}$, all zeros of multiplicity at least $k$. Suppose that there exists a number $A \geq 1$ such that $\left|f^{(k)}(z)\right| \leq A$ whenever $f \in \mathcal{F}$ and $f=0$. If $\mathcal{F}$ is not normal in $\Delta$, then for $0 \leq \alpha \leq k$, there exist

1. a number $r \in(0,1)$;

2. a sequence of complex numbers $z_{n},\left|z_{n}\right|<r$;

3. a sequence of functions $f_{n} \in \mathcal{F}$;

4. a sequence of positive numbers $\rho_{n} \rightarrow 0^{+}$;

such that $g_{n}(\xi)=\rho_{n}^{-\alpha} f_{n}\left(z_{n}+\rho_{n} \xi\right)$ locally uniformly (with respect to the spherical metric) to a nonconstant meromorphic function $g(\xi)$ on $\mathrm{C}$, and moreover, the zeros of $g(\xi)$ are of multiplicity at least $k, g^{\sharp}(\xi) \leq g^{\sharp}(0)=k A+1$. In particular, $g$ has order at most 2.

Here, as usual, $g^{\sharp}(\xi)=\frac{\left|g^{\prime}(\xi)\right|}{1+|g(\xi)|^{2}}$ is the spherical derivative.

Lemma 2.2([1]). Let $f(z)$ be a transcendental meromorphic function of finite order and $k$ be a positive integer, let a be a non-zero finite complex number. If all zeros of $f(z)$ are of multiplicity at least $k+1$, then $f^{(k)}(z)$ assume a infintely often.

Lemma 2.3([8]). Let $f(z)=a_{n} z^{n}+a_{n-1} z^{n-1}+, \ldots,+a_{0}+\frac{q(z)}{p(z)}$, where $a_{0}, a_{1}, \ldots, a_{n}$ are constants with $a_{n} \neq 0$, and $q(z)$ and $p(z)$ are co-prime polynomials with $\operatorname{deg} q(z)<\operatorname{deg} p(z) ;$ and let $k$ be a positive integer. If $f^{(k)}(z) \neq 1$, then

$$
f(z)=\frac{z^{k}}{k !}+, \ldots,+a_{0}+\frac{1}{a z+b},
$$

where $a(\neq 0), b, a_{0}, \ldots$ are constants.

\section{Proof of Theorem 1 and Theorem 2}

Proof of Theorem 1. We may assume that $S=\{a, b\}$, where $a$ and $b$ are two distinct constants and $D=\Delta=\{|z|<1\}$, the unit disk. Now we consider two cases

Case 1. $a b \neq 0$. Suppose that $\mathcal{F}$ is not normal in $D=\Delta$. Withoutloss of generality, we assume that $\mathcal{F}$ is not normal at $z_{0}=0$. Then, by Lemma 2.1, there exist 
1. a number $r \in(0,1)$;

2. a sequence of complex numbers $z_{j},\left|z_{j}\right|<r$;

3. a sequence of functions $f_{j} \in \mathcal{F}$;

4. a sequence of positive numbers $\rho_{j} \rightarrow 0^{+}$

such that $g_{j}(\xi)=\frac{f_{j}\left(z_{j}+\rho_{j} \xi\right)}{\rho_{j}^{k}}$ converges uniformly with respect to the spherical metric to a non-constant mermorphic functions $g(\xi)$ in $C$. Moreover, $g(\xi)$ is of order at most 2, all of whose zeros have multiplicity at least $k+1$. Set $Q(w)=$ $w^{q}+a_{q-1}(0) w^{q-1}+, \ldots,+a_{1}(0) w$.

We claim that

(i) $Q\left(g^{(k)}\right) \neq a$,

(ii) $Q\left(g^{(k)}\right) \neq b$.

Suppose now that $Q\left(g^{(k)}\left(\xi_{0}\right)\right)=a$. We claim that $Q\left(g^{(k)}\right) \not \equiv a$. Otherwise, from the definition of $Q(w)$, there exist a nonzero constant $h$ such that $g^{(k)}(\xi) \equiv h, g$ must be a polynomial of at most degree $k$, which contradicts the fact that each zero of $g(\xi)$ are of multiplicity at least $k+1$. Since $Q\left(g^{(k)}\left(\xi_{0}\right)\right)=a$. Obviously, $g\left(\xi_{0}\right) \neq \infty$. Hence there exists $\delta>0$ such that $g(\xi)$ is analytic on $G_{2 \delta}=\left\{\xi:\left|\xi-\xi_{0}\right|<2 \delta\right\}$. Thus $g_{j}^{(i)}(\xi)(i=0,1,2, \ldots, k)$ are analytic on $G_{\delta}=\left\{\xi:\left|\xi-\xi_{0}\right|<\delta\right\}$ for large $j$ and $g_{j}^{(i)}(\xi)$ converges uniformly to $g^{(i)}(\xi)(i=0,1,2, \ldots, k)$ on $\bar{G}_{\delta}=\left\{\xi:\left|\xi-\xi_{0}\right| \leq \delta\right\}$.

As

$$
G\left(f_{j}\right)\left(z_{j}+\rho_{j} \xi\right)-a=P\left(f_{j}^{(k)}\left(z_{j}+\rho_{j} \xi\right)\right)+H\left(f_{j}, f_{j}^{\prime}, \ldots, f_{j}^{(k)}\right)\left(z_{j}+\rho_{j} \xi\right)-a,
$$

and

$$
H\left(f_{j}, f_{j}^{\prime}, \ldots, f_{j}^{(k)}\right)\left(z_{j}+\rho_{j} \xi\right)=\sum_{i=1}^{n} b_{i}\left(z_{j}+\rho_{j} \xi\right) \rho_{j}^{(k+1) \gamma_{M_{i}}-\Gamma_{M_{i}}} M_{i}\left(g_{j}, g_{j}^{\prime}, \ldots, g_{j}^{(k)}\right)(\xi) .
$$

Considering $b_{i}(z)$ are analytic on $\mathrm{D}(i=1,2, \ldots, n)$, we have

$$
\left|b_{i}\left(z_{j}+\rho_{j} \xi\right)\right| \leq M\left(\frac{1+r}{2}, b_{i}(z)\right)<\infty,(i=1,2, \ldots, n)
$$

for sufficiently large $j$.

Hence we deduce from $\left.\frac{\Gamma}{\gamma}\right|_{H}<k+1$ that

$$
\sum_{i=1}^{n} b_{i}\left(z_{j}+\rho_{j} \xi\right) \rho_{j}^{(k+1) \gamma_{M_{i}}-\Gamma_{M_{i}}} M_{i}\left(g_{j}, g_{j}^{\prime}, \ldots, g_{j}^{(k)}\right)(\xi)
$$

converges uniformly to 0 on $D_{\frac{\delta}{2}}=\left\{\xi:\left|\xi-\xi_{0}\right|<\frac{\delta}{2}\right\}$.

Thus we know that $G\left(f_{j}\right)\left(z_{j}+\rho_{j} \xi\right)-a$ converges uniformly to $Q\left(g^{(k)}\right)-a$ on $D_{\frac{\delta}{2}}=\left\{\xi:\left|\xi-\xi_{0}\right|<\frac{\delta}{2}\right\}$. 
Hence, by Hurwitz's theorem we deduce that there exist $\xi_{j}, \xi_{j} \rightarrow \xi_{0}$ such that, for large $j$,

$$
P\left(g_{j}^{(k)}\left(\xi_{j}\right)\right)+\sum_{i=1}^{m} b_{i}\left(z_{j}+\rho_{j} \xi_{j}\right) \rho_{j}^{(k-1) n_{1}+, \ldots,+n_{k-1}} M_{i}\left(g, g_{j}^{\prime}, \ldots, g_{j}^{(k)}\right)\left(\xi_{j}\right)=a,
$$

thus

$$
P\left(f_{j}^{k}\left(z_{j}+\rho_{j} \xi_{j}\right)\right)+H\left(f_{j}, f_{j}^{\prime}, \ldots, f_{j}^{(k)}\right)\left(z_{j}+\rho_{j} \xi_{j}\right)=a .
$$

It follows from $G(f)=a \Rightarrow f=a$ or $b$ that $f_{j}\left(z_{j}+\rho_{j} \xi_{j}\right)=a$ or $b$.

Thus we have $g\left(\xi_{0}\right)=\lim _{j \rightarrow \infty} g_{j}\left(\xi_{j}\right)=\infty$, which contradicts $Q\left(g^{(k)}\left(\xi_{0}\right)\right)=a$.

In a similar fashion, we can prove that $Q\left(g^{(k)}\right)\left(\xi_{0}\right) \neq b$. This completes the proof of (i) and (ii).

By (ii), it follows $Q\left(g^{(k)}\right) \neq b$ and the definition of $Q(w)$ that there exist a non-zero constant $d$ satisfying $g^{(k)} \neq d$. By Lemma 2.2, we know $g$ is not a transcendental meromorphic function. Since $g$ has zeros of multiplicity at least $k+1$ and $g^{(k)} \neq d$, it follows that $g$ is not a polynomial. Hence by Lemma 2.2, we obtain that $g(\xi)=\frac{d \xi^{k}}{k !}+\cdots+a_{0}+\frac{1}{A \xi+B}$, where $B, A(\neq 0), a_{0}, \ldots$ are constants. Thus

$$
g^{(k)}(\xi)=d+\frac{(-1)^{k} k ! A^{k}}{(A \xi+B)^{k+1}} .
$$

It follows that $g^{(k)}(\xi)=h$ has solutions, So $Q\left(g^{(k)}\right)=a$ has solutions, which contradicts (i). Hence $\mathcal{F}$ is normal in $D$.

Case 2. $a b=0$. We may assume that $b=0$. Suppose that $\mathcal{F}$ is not normal in $D=\Delta$. Withoutloss of generality, we assume that $\mathcal{F}$ is not normal at $z_{0}=0$. Then, by Lemma 2.1, there exist

1. a number $r \in(0,1)$;

2. a sequence of complex numbers $z_{j},\left|z_{j}\right|<r$;

3. a sequence of functions $f_{j} \in \mathcal{F}$;

4. a sequence of positive numbers $\rho_{j} \rightarrow 0^{+}$

such that $g_{j}(\xi)=\frac{f_{j}\left(z_{j}+\rho_{j} \xi\right)}{\rho_{j}^{k}}$ converges uniformly with respect to the spherical metric to a non-constant mermorphic functions $g(\xi)$ in $C$. Moreover, $g(\xi)$ is of order at most 2, all of whose zeros have multiplicity at least $k+1$. Set $Q(w)=$ $w^{q}+a_{q-1}(0) w^{q-1}+, \ldots,+a_{1}(0) w$.

We claim that

(iii) $Q\left(g^{(k)}\right) \neq a$,

(iv) $Q\left(g^{(k)}\right)=0 \Leftrightarrow g=0$.

Now we prove (iii). Suppose now that $Q\left(g^{(k)}\left(\xi_{0}\right)\right)=a$. We claim that $Q\left(g^{(k)}\right) \not \equiv$ $a$. Otherwise, from the definition of $Q(w)$, there exist a nonzero constant $h$ such that $g^{(k)}(\xi) \equiv h, g$ must be a polynomial of at most degree $k$, which contradicts the fact that each zero of $g(\xi)$ are of multiplicity at least $k+1$. Since $Q\left(g^{(k)}\left(\xi_{0}\right)\right)=a$. 
Suppose $Q\left(g^{(k)}\left(\xi_{0}\right)\right)=a$, by Hurwitz's theorem, we know that there exists $\left\{\xi_{j}\right\}$, $\xi_{j} \rightarrow \xi_{0}$, such that, for $j$ sufficiently large, and the similarly proof about case 1 , we obtain $G\left(f_{j}\right)\left(z_{j}+\rho_{j} \xi_{j}\right)$ converges uniformly to $Q\left(g^{(k)}\right)$ on $D_{\frac{\delta}{2}}=\left\{\xi:\left|\xi-\xi_{0}\right|<\frac{\delta}{2}\right\}$. So

$$
G\left(f_{j}\left(z_{j}+\rho_{j} \xi_{j}\right)\right)=a .
$$

It now follows from $G(f)=a \Rightarrow f=a$ or 0 that $f_{j}\left(z_{j}+\rho_{j} \xi_{j}\right)=a$ or 0 . If $f_{j}\left(z_{j}+\rho_{j} \xi_{j}\right)=a$, then

$$
g_{j}\left(\xi_{j}\right)=\frac{f_{j}\left(z_{j}+\rho_{j} \xi_{j}\right)}{\rho_{j}^{k}}=\frac{a}{\rho_{j}^{k}} .
$$

So $g\left(\xi_{0}\right)=\lim _{j \rightarrow \infty} g_{j}\left(\xi_{j}\right)=\infty$, which contradicts $Q\left(g^{(k)}\left(\xi_{0}\right)\right)=a$. If $f_{j}\left(z_{j}+\rho_{j} \xi_{j}\right)=0$, then $Q\left(g^{(k)}(\xi)\right)=a \Rightarrow g=0$. From the definition of $Q(w)$, similarly the proof of case 1 , there exists a nonzero constant $h$ such that $g^{(k)}(\xi)=h$. So all zeros of $g^{(k)}(\xi)=h$ are the zeros of $g=0$. By the all zeros of $g$ have multiplicity at least $k+1$, we know all zeros of $g$ are zeros of $g^{(k)}$. So all zeros of $g^{(k)}(\xi)=h$ are the zeros of $g^{(k)}(\xi)=0$, we deduce $h=0$, a contradiction.

Next, we prove(iv). Suppose now that $Q\left(g^{(k)}\left(\xi_{0}\right)\right)=0$. We claim that $Q\left(g^{(k)}\right) \not \equiv 0$. Otherwise, from the definition of $Q(w)$, we get $g^{(k)}(\xi) \equiv 0, g$ must be a polynomial of at most degree $k-1$, which contradicts the fact that each zero of $g(\xi)$ are of multiplicity at least $k+1$. Since $Q\left(g^{(k)}\left(\xi_{0}\right)\right)=0$. By Hurwitz's theorem, we know that there exists $\left\{\xi_{j}\right\}, \xi_{j} \rightarrow \xi_{0}$, such that, for $j$ sufficiently large, and the similarly above proof, we obtain $G\left(f_{j}\left(z_{j}+\rho_{j} \xi_{j}\right)\right)=0$. It now follows from $G(f)=0 \Rightarrow f=a$ or 0 that $f_{j}\left(z_{j}+\rho_{j} \xi_{j}\right)=a$ or 0 . If $f_{j}\left(z_{j}+\rho_{j} \xi_{j}\right)=a$, then $g\left(\xi_{0}\right)=\lim _{j \rightarrow \infty} g_{j}\left(\xi_{j}\right)=\infty$, which contradicts $Q\left(g^{(k)}\left(\xi_{0}\right)\right)=0$.

Hence $f_{j}\left(z_{j}+\rho_{j} \xi_{j}\right)=0$, so that $g\left(\xi_{0}\right)=\lim _{j \rightarrow \infty} g_{j}\left(\xi_{j}\right)=0$. Thus we deduce that $Q\left(g^{(k)}\right)=0 \Rightarrow g=0$. Obviously, $g=0 \Rightarrow g^{(k)}=0 \Rightarrow Q\left(g^{(k)}\right)=0$. This proves (iv).

By (iii), it follows $Q\left(g^{(k)}\right) \neq a$ and the definition of $Q(w)$ that there exist a non-zero constant $h$ satisfying $g^{(k)} \neq h$. By Lemma 2.2, We know $g$ is not a transcendental meromorphic function. Since $g$ has zeros of multiplicity at least $k+1$ and $g^{(k)} \neq h$, it follows that $g$ is not a polynomial. Hence by Lemma 2.3, we obtain that $g(\xi)=\frac{h \xi^{k}}{k !}+\cdots+a_{0}+\frac{1}{A \xi+B}$, where $B, A(\neq 0), a_{0}, \ldots$ are constants. By the condition that $g$ has zeros of multiplicity at least $k+1$, thus $g(\xi)$ has only a zero. On the other hand, $g^{(k)}(\xi)=h+\frac{(-1)^{k} k ! A^{k}}{(A \xi+B)^{k+1}}$. Obviously, $g^{(k)}=0$ has $k+1$ distinct solutions, which contradicts $g^{(k)}=0 \Rightarrow Q\left(g^{(k)}\right)=0 \Rightarrow g=0$. Hence $\mathcal{F}$ is normal in D. The proof of Theorem 1.1 is complete.

Proof of theorem 2. We may assume that $S_{1}=\{a, b\}, S_{2}=\{c\}$ where $a, b, c$ are three distinct constants and $D=\Delta=\{|z|<1\}$. Suppose that $\mathcal{F}$ is not normal in $\Delta$. Then by Lemma 2.1, we can find $f_{j} \in \mathcal{F}, z_{j} \in \Delta$ and $\rho_{j} \rightarrow 0^{+}$such that $g_{j}(\xi)=f_{j}\left(z_{j}+\rho_{j} \xi\right)$ converges locally uniformly with respect to the spherical metric 
to a nonconstant meromorphic function $g$ on $C$, all of whose zeros have multiplicity at least $k$.

We claim that

(i) $g^{(k)} \neq 0$,

(ii) $g \neq a, g \neq b$, and $g \neq c$.

Suppose now that $g^{(k)}\left(\left(\xi_{0}\right)\right)=0$. Obviously, $g\left(\xi_{0}\right) \neq \infty$. We claim that $g^{(k)} \not \equiv 0$. Otherwise, $g$ must be a polynomial of degree less than $k$, which contradicts the fact that each zero of $g(\xi)$ are of multiplicity at least $k$. Similarly above the proof of theorem 1 , there exist a constant $h^{\prime}$ satisfying $g^{(k)}=h^{\prime} \Rightarrow P\left(g^{(k)}\right)=a$, and $g_{j}^{(k)}(\xi)-\rho_{j}^{k} h^{\prime} \rightarrow g^{(k)}(\xi)$ on a neighborhood of $\xi_{0}$; also there exists $\xi_{j}, \xi_{j} \rightarrow \xi_{0}$, such that (for $j$ sufficiently large)

$$
0=g^{(k)}\left(\xi_{0}\right)=g_{j}^{(k)}\left(\xi_{j}\right)-\rho_{j}^{k} h^{\prime}=\rho_{j}^{k}\left(f_{j}^{(k)}\left(z_{j}+\rho_{j} \xi_{j}\right)-h^{\prime}\right) .
$$

Thus $f_{j}^{(k)}\left(z_{j}+\rho_{j} \xi_{j}\right)=h^{\prime}$. So we obtain $P\left(f_{j}^{(k)}\left(\xi_{j}\right)\right)=a$.

It follows from $P\left(f^{(k)}\right)=a \Rightarrow f=a$ or $b$ that $f_{j}\left(z_{j}+\rho_{j} \xi_{j}\right)=a$ or $b$, and so $g\left(\xi_{0}\right)=\lim _{j \rightarrow \infty} g_{j}\left(\xi_{j}\right)=a$ or $b$. In a similar fashion, there exist a constant $p$ satisfying $g^{(k)}=p \Rightarrow P\left(g^{(k)}\right)=c$. Using $g_{j}^{(k)}(\xi)-\rho_{j}^{k} p$, and the similarly above the proof, we obtain that $g\left(\xi_{0}\right)=c$, which contradicts $a \neq c$ and $b \neq c$. This completes the proof of (i).

Next, we prove (ii). Suppose that $g\left(\xi_{0}\right)=a$. Then there exists $\xi_{j} \rightarrow \xi_{0}$, such that(for $j$ sufficiently large)

$$
a=g\left(\xi_{0}\right)=g_{j}\left(\xi_{j}\right)=f_{j}\left(z_{j}+\rho_{j} \xi_{j}\right) .
$$

Thus $P\left(f_{j}^{(k)}\right)=a$ or $b$. So there exist two constants $h^{\prime}$ and $d$ such that $g_{j}^{(k)}\left(\xi_{j}\right)=h^{\prime}$ or $d$. Therefore, $g^{(k)}\left(\xi_{0}\right)=\lim _{j \rightarrow \infty} \rho_{j}^{k} g_{j}^{(k)}\left(\xi_{j}\right)=0$, which contradicts (i).

In a similar fashion, we obtain that $g \neq b$ and $g \neq c$.

Now by Picard's Theorem and (ii), $g$ is a constant, a contradiction. Thus $\mathcal{F}$ is normal in D. Theorem is proved.

Acknowledgements This paper is supported by project 10XKJ01 from Leading Academic Discipline Project of Shanghai Dianji University, and also supported by the Key Development Project 12C104 from Shanghai Dianji University.

\section{References}

[1] W. Bergweiler and A. Eremenko, On the singularities of the inverse to a meromorphic function of finite order, Rev. Mat. Iber., 11(1995), 355-373. 
[2] M. L. Fang and L. Zalcman, Normal families and shared values of meromorphic functions III, Comput. Methods Funct. Theory, 2(2002), 385-395.

[3] W. K. Hayman, Meromorphic Functions, Clarendon Press, Oxford, 1964.

[4] C. L. Lei, M. L. Fang and D. G. Yang, Normal of meromorphic functions and shared sets, Advance in Inequalities for Series., (2008), 155-162.

[5] X. C. Pang and L. Zalcman, Normality and shared values, Ark. Mat., 38(2000), 171182.

[6] X. C. Pang and L. Zalcman, Normal families and shared values, Bull. London Math. Soc., 32 (2000), 325-331.

[7] W. Schwick, Sharing values and normality, Arch Math., 59(1992), 50-54.

[8] Y. F. Wang and M. L. Fang, Picard values and normal families of meromorphic functions with zeros, Acta Math., Sinica, New Series, 14(1)(1998), 17-26.

[9] L. Zalcman, Normal families new perspectives, Bull. Amer. Math. Soc., 35(1998), 215-230. 